\title{
Oxidative Stress and Heme Oxygenase-1 Regulated Human Mesenchymal Stem Cells Differentiation
}

\author{
Luca Vanella, ${ }^{1}$ Christopher Sanford Jr., ${ }^{2}$ Dong Hyun Kim, ${ }^{3}$ \\ Nader G. Abraham, ${ }^{3}$ and Nabil Ebraheim ${ }^{2}$ \\ ${ }^{1}$ Department of Biological Chemistry, Medical Chemistry and Molecular Biology, University of Catania, Viale Andrea Doria 6, \\ 95125 Catania, Italy \\ ${ }^{2}$ Department of Orthopedic Surgery, University of Toledo College of Medicine, Toledo, OH 43614, USA \\ ${ }^{3}$ Department of Physiology and Pharmacology, University of Toledo College of Medicine, Toledo, OH 43614, USA
}

Correspondence should be addressed to Luca Vanella, vanella@hotmail.it

Received 21 September 2011; Revised 9 November 2011; Accepted 14 November 2011

Academic Editor: David E. Stec

Copyright ( $) 2012$ Luca Vanella et al. This is an open access article distributed under the Creative Commons Attribution License, which permits unrestricted use, distribution, and reproduction in any medium, provided the original work is properly cited.

\begin{abstract}
This paper describes the effect of increased expression of HO-1 protein and increased levels of HO activity on differentiation of bone-marrow-derived human MSCs. MSCs are multipotent cells that proliferate and differentiate into many different cell types including adipocytes and osteoblasts. HO, the rate-limiting enzyme in heme catabolism, plays an important role during MSCs differentiation. HO catalyzes the stereospecific degradation of heme to biliverdin, with the concurrent release of iron and carbon monoxide. Upregulation of HO-1 expression and increased $\mathrm{HO}$ activity are essential for MSC growth and differentiation to the osteoblast lineage consistent with the role of HO-1 in hematopoietic stem cell differentiation. HO-1 participates in the MSC differentiation process shifting the balance of MSC differentiation in favor of the osteoblast lineage by decreasing PPAR $\gamma$ and increasing osteogenic markers such as alkaline phosphatase and BMP-2. In this paper, we define HO-1 as a target molecule in the modulation of adipogenesis and osteogenesis from MSCs and examine the role of the HO system in diabetes, inflammation, osteoporosis, hypertension, and other pathologies, a burgeoning area of research.
\end{abstract}

\section{Background}

1.1. Mesenchymal Stem Cells. Among cells bone-marrowderived MSCs have attracted a great deal of attention in the past decade because of their high versatility. MSCs have shown promise in the treatment of cardiovascular disease in a series of animal models. Despite being a rare cell population, MSCs can be extensively expanded in vitro thus making them of potential use in the clinic [1]. Human MSCs derived from bone marrow are multipotent cells that differentiate and proliferate into many different cell types in various tissues [1-3]. Bone marrow mononuclear cells can be isolated with ease with a Ficoll-Paque PLUS density gradient. Human MSCs give rise to both osteoblastic and adipogenic lineages when cultured with specific differentiation media. The adipogenic media comprise of complete culture medium supplemented with DMEM-high glucose, FBS, insulin, dexamethasone, and indomethacin. The osteogenic media contain ascorbic acid (for appropriate collagen and extracellular matrix production) and $\beta$-glycerophosphate (for appropriate mineralization). Several studies demonstrated that an increase in the ROS leads to the elevation of the levels of inflammatory cytokines in adipose tissue. Oxidative stress is a major factor impairing MSCs function resulting in decreased osteogenesis in favor of adipogenesis. Whether MSCs differentiate into osteoblasts or adipocytes is due to multiple signaling pathways including those heavily influenced by $\mathrm{HO}-1$ and -2 [4].

1.2. Role of HO during Bone Formation. Recent studies have shown that several growth factors including OGP enhance differentiation of MSCs to osteoblasts [5] and that EGF and OGP signaling pathways enhance osteoblast cell proliferation. Osteogenic growth peptide is a naturally occurring tetradecapeptide that is both an anabolic agent and a hematopoietic stimulator [6]. For example, OGP increases 
osteoblast proliferation, AP activity, and matrix synthesis and mineralization. It prevents glucocorticoid-induced apoptosis and the subsequent bone remodeling alterations that are associated with steroids [7]. Thus, role of $\mathrm{HO}$ in the fluctuations of ROS and its effect on osteonectin levels and in MSC-derived osteoblasts will be described. Cytokines and HO activity have a regulatory role in MSCs microenvironment and hematopoiesis [8]. HO attenuates the overall production of ROS through its ability to degrade the prooxidant, heme, resulting in the production of carbon monoxide, biliverdin/bilirubin, and the release of free iron. These three products of heme degradation play an important role in signaling cascades, cell proliferation and differentiation. $\mathrm{HO}$ is the enzyme that catalyzes the rate-limiting step in the degradation of heme and exists in two forms: the inducible HO- 1 form and the constitutive HO-2 form [4]. During fracture repair, activation of hypoxia-inducible factor (HIF)- 1 and its target genes, VEGF and HO-1, regulate bone remodeling. Bone remodeling is a physiological process which includes bone resorption (by osteoclasts) and bone formation (by the osteoblasts) and requires coordination of three cell types, osteocytes, osteoblasts, and osteoclasts. During aging and in several pathologies, the rate of bone turnover increases, but this is characterized by an impaired osteoblastic bone formation compared to osteoclastic bone resorption caused by decreased number and activity of osteoblastic cells (Figure 1) [9-11].

This association suggests a role of HO- 1 in bone metabolism. As previously stated, $\mathrm{HO}$ has strong implications in bone marrow stem cell differentiation $[8,12]$. Most notably, HO-1 expression is increased during osteoblast stem cell development. This increase in HO-1 expression precedes an increase in alkaline phosphatase, bone morphogenic protein, osteonectin, and RUNX-2 mRNA [13]. The function of bone-specific alkaline phosphatase has been shown to be that of a biochemical indicator of bone turnover. Upregulation of HO-1 increased MSC-mediated osteoblasts with an associated decrease in adipocytes. OGP increased HO1 levels [13]. OGP also lead to an increase in pAKT, an antiapoptotic protein, as well as an increase in eNOS and p-eNOS (Figure 2) [13]. Past research has demonstrated that eNOS is an enzyme expressed in osteoblasts that, when deficient, has been shown to lead to a significant reduction in bone formation in murine models [14]. Both eNOS and $\mathrm{NO}$ are stimulators of BMP-2 and increase differentiation of osteoblasts $[15,16]$. Increased HO-1 expression has also been shown to increase alkaline phosphatase as well as DNA accumulation and mineralization when compared to osteoblasts not treated with OGP [13]. Osteoblasts cultured in hyperglycemic conditions showed reduced levels of bone BMP-2, osteonectin, pAMPK (a signaling molecule in osteoblasts), and eNOS. The reduction of these osteogenic proteins and enzymes was reversed by OGP which upregulated HO-1 with a subsequent increase in BMP-2, HO-1, eNOS, and pAMPK [13]. HO-1 expression and activity are essential for osteoblast differentiation from MSCs. Although basal levels of HO- 1 and $\mathrm{HO}$ activity are necessary for osteoblast growth, an increase in HO-1 amplifies osteoblast differentiation. Additionally, HO-1 is required to increase pAKT, pAMPK, peNOS levels, and NO bioavailability $[17,18]$.

\section{HO-1 and Oxidative Stress}

An additional important association of $\mathrm{HO}-1$ is this enzyme's influence on oxidative stress and reactive oxygen species (ROS). Hyperglycemia and certain cytokines result in an increase in ROS. HO-1 is inhibited in the presence of high glucose. High glucose suppressed HO-1 expression in both cell lines [19-21] and animal models [22-24].

Intracellularly, reduction-oxidation homeostasis is maintained by the balance between oxidants and antioxidants. Antioxidants including $\mathrm{HO}$, superoxide dismutase, glutathione peroxidase, and catalase are endogenous. Exogenous antioxidants are often derived from food and include vitamins A, C, E, selenium, resveratrol, $\alpha$-tocopherol, and $\beta$ carotene [25-27]. When the natural balance between oxidants and antioxidants is altered, ROS can potentially damage cellular structures like DNA, proteins, and phospholipids. This process, called oxidative stress, is implicated in several neurodegenerative and metabolic diseases including obesity and type 2 diabetes. Moderate-to-severe obesity is associated with an increased risk for hypertension and insulin resistance in humans and animals $[22,28,29]$. The antioxidant effects of $\mathrm{HO}$ arise from its ability to degrade heme from destabilized heme proteins and from the production of biliverdin and bilirubin, products of $\mathrm{HO}$ with potent antioxidant properties. Heme is a prooxidant so, therefore, its breakdown is antioxidative. Although there appears to be a convincingly clear link between HO1 and apoptosis, the specific mechanism through which HO-1 prevents apoptosis remains unclear [21, 30, 31]. It is likely that part of the anti-apoptotic role of HO-1 is based on its function as an antioxidant enzyme. However, the possibility remains that one or more of its products play additional roles in anti-apoptotic mechanisms. This association between a reduction in ROS with an increase in HO-1 expression was demonstrated by treating MSCs with CoPP, a strong inducer of HO-1. Exposure of the MSCs to CoPP is effective in decreasing ROS while high glucose concentrations increase ROS. A reduction in ROS permits the restoration of osteoblast markers, specifically induction of osteoprotegerin and osteocalcin [4].

The discovery that inhibition of HO-1 expression shifts mesenchymal stem cells to favor adipocytic cells at the expense of osteoblastic cells $[4,13]$ demonstrates that high glucose has an adipogenic potential and also that a direct link is present between $\mathrm{HO}-1$ suppression and an increase in adipogenesis [13]. The exact mechanisms through which HO-1 affects adipocyte and osteoblast differentiation and protects against oxidative injury remain unclear. However, targeting $\mathrm{HO}-1$ expression is a gateway to increasing osteoblast stem cell differentiation, decreasing oxidative stress, and to the attenuation of osteoporosis through the promotion of bone formation. 


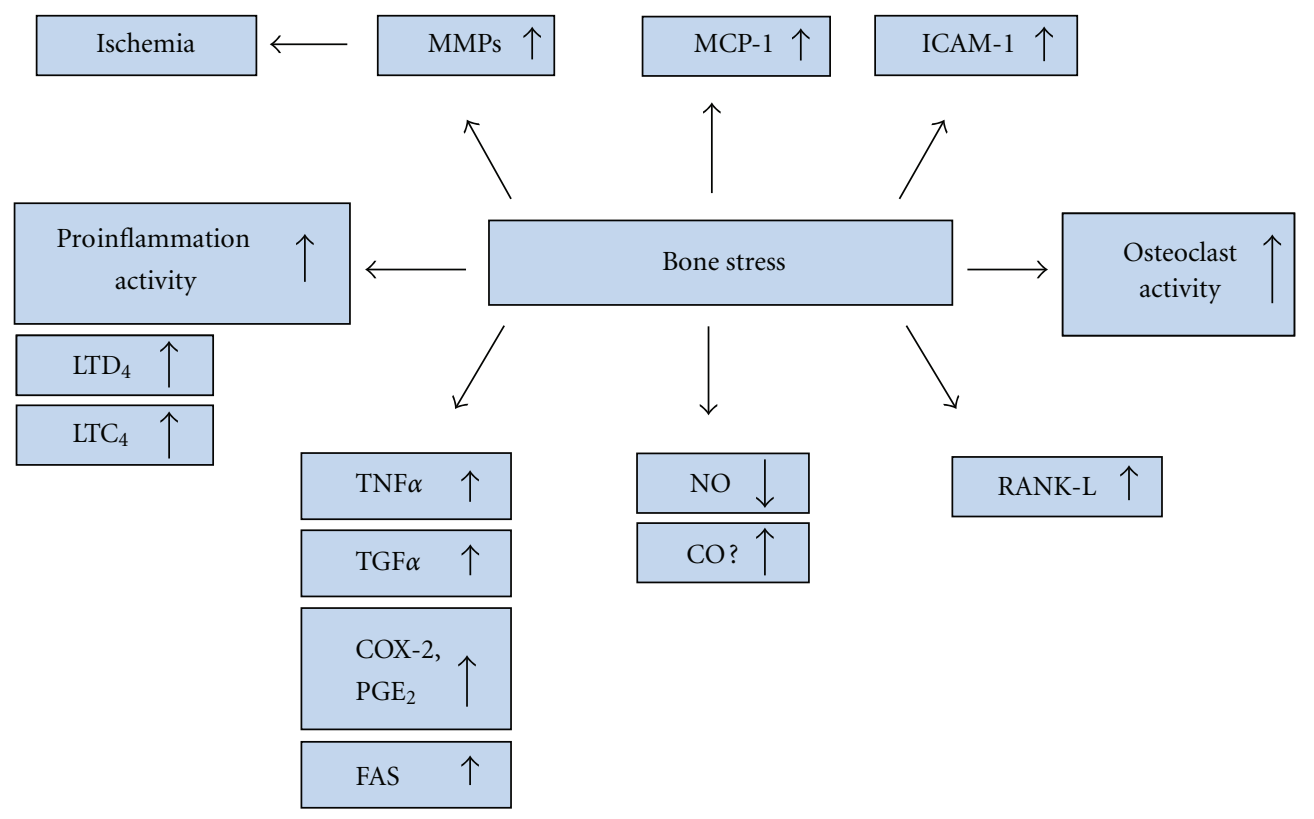

FIGURE 1: Diagram representing the adverse effects of stress on bone, leading to the releases of inflammatory molecules.

2.1. Introduction of PPAR $y$ and TZD. There appears to be a strong link between HO-1 and PPAR $\gamma$. PPAR $\gamma$ has been found to increase with the suppression of HO-1 [13]. The increase in PPAR $y$ is associated with a reduction of HO- 1 expression and coupled to a significant increase in adipogenesis [13]. PPAR $\gamma$ is a transcription factor that has been implicated in the development of osteoporosis. PPAR $\gamma$ is considered to be the master regulator of adipogenesis $[32,33]$, and its expression is believed to increase with age and in diabetics. PPAR $y$ is abundantly expressed in mature adipocytes. Its levels are also increased in the livers of animals that have developed steatosis [34, 35]. Loss of the gene that encodes for PPAR $\gamma$ in embryonic fibroblasts results in the complete absence of adipogenesis in murine studies [36]. In addition, adipocyte differentiation can be stimulated by ectopic expression and activation of PPAR $\gamma$. Conversely, the absence of this gene results in an increase in osteoblasts and bone mass [37]. PPAR $y$ is expressed in human MSCs [38-40]. This evidence coupled with the fact that bone marrow fat increases and bone marrow osteoblasts decrease with age in both animals and humans $[41,42]$ demonstrates a possible strong link between PPAR $y$ and osteoporosis. Furthermore, PPAR $y$ has been found to decrease the levels of core-binding factor alpha (Cbfa1) and RUNX-2, two factors needed for osteoblast development. By leading to the differentiation of adipocytes over osteoblasts, activation of PPAR $\gamma$ subsequently leads to a decrease in bone mineral density and appears to be strongly correlated to the development and progression of osteoporosis. The stimulation of PPAR $\gamma$ occurs following upregulation of PPAR $\gamma$ expression by TZD [43]. TZDs provide diabetics with many benefits including lower blood glucose levels and reduced rates of atherosclerosis. However, these benefits come with multiple negative effects on bone such as inhibiting osteoblast formation [44-46] and inducing apoptosis of mature osteoblasts. Activation of PPAR $\gamma$ by TZDs also results in the suppression of many vital osteogenic transcription factors in both animal models and humans $[47,48]$. The end result of these antiosteogenic effects is a decrease in bone mass. Furthermore, TZD use has been shown to increase the risk of fracture in postmenopausal women [49-51]. A large cohort study showed that the use of TZDs in type 2 diabetics increased the fracture risk in women over 65 years old. This increased fracture risk was seen after only one year of TZD use [51]. The association between TZD and fracture risk is likely due to a discrepancy between bone formation and resorption as rosiglitazone, a thiazolidinedione, has been found to decrease bone mass in the hip and decrease serum osteocalcin levels [52]. Aging leads to a decrease in HO-1 and an increase in bone marrow adiposity with bone loss and an increase in the expression of PPAR $\gamma$ and, ultimately, PPAR $\gamma$ [53]. Extensive research demonstrates that PPAR $\gamma$ plays a pivotal role in regulating MSC specification towards adipogenesis versus osteogenesis and is instrumental in governing both bone mineral density and osteoporosis.

2.2. HO-1 and CYP-450. HO-1 induction increased the levels of cytochrome P450- (P450-) derived epoxyeicosatrienoic acids (EETs), which further decreased PPAR $\gamma$ but increased osteoblasts. We examined if EET levels regulate the MSCderived adipocytes. Expression of FAS and PPAR $\gamma$ levels was significantly $(P<0.05)$ increased in preadipocyte (14 days of MSC-derived adipocyte differentiation), and conversely pACC and $\beta$-catenin were decreased $(P<$ $0.05)$ in preadipocyte. The increase in FAS and PPAR $\gamma$ in preadipocyte was suppressed by the EET. In contrast, the EET treatments significantly increased both pACC and $\beta$ catenin compared to cell treated with vehicle solutions. 

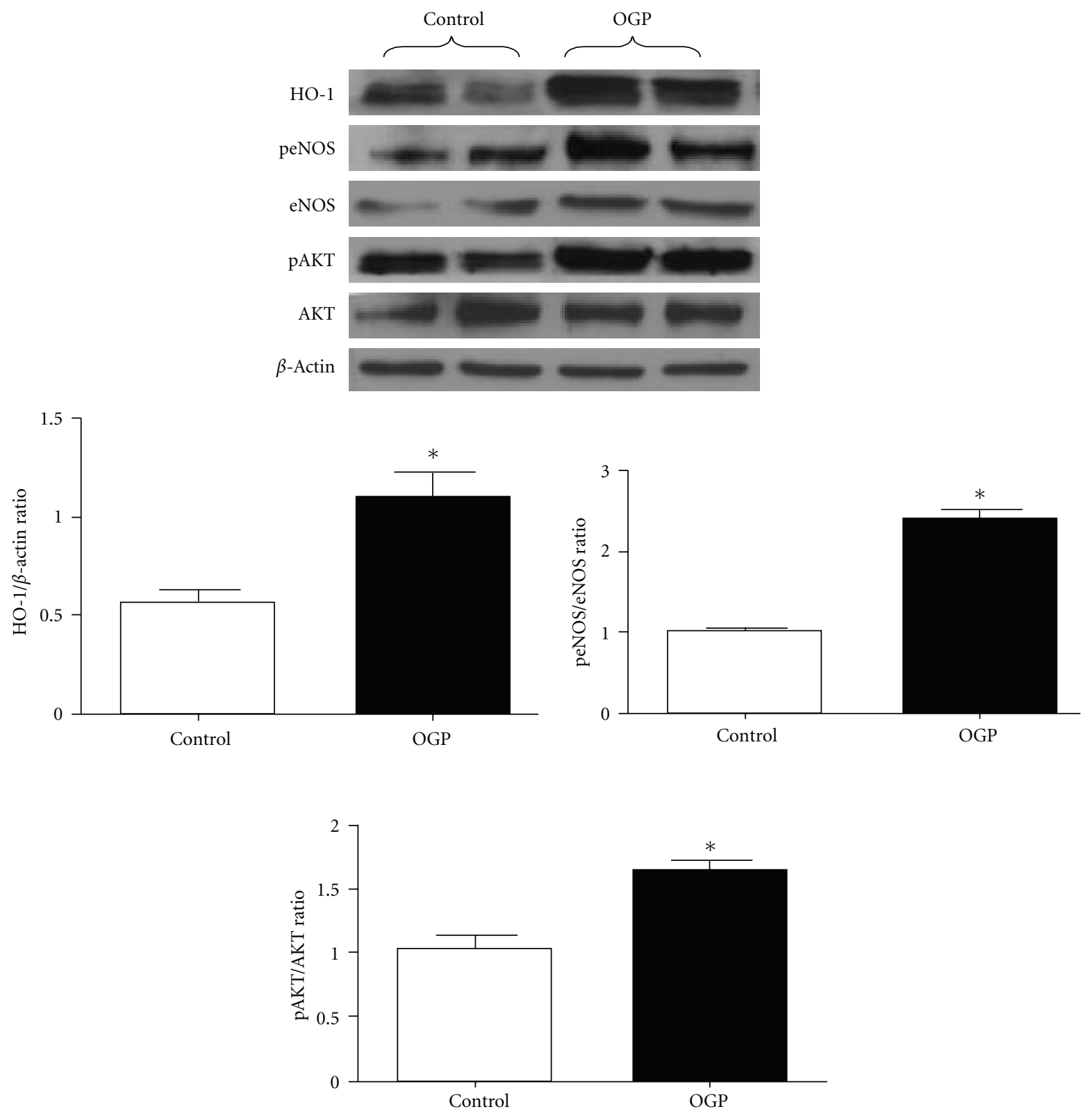

FIGURE 2: Effect of OGP on HO-1, peNOS/eNOS, and pAKT/AKT proteins expression after 21 days of osteoblast differentiation. Quantitative densitometry evaluation of the proteins ratio was determined. Data are expressed as means \pm SEM of three independent experiments; ${ }^{*} P<0.05$ control versus OGP.

Further, MSCs-derived adipocyte exhibited a significantly higher expression of PPAR $\gamma$, SREBP-1, and GLUT4 compared to MSCs-adipocyte grown in the presence of EET [54]. Adipocyte cultured in the absence of the EET agonist showed HO-1 levels that were decreased at day 10 and day 15 compared to adipocyte cultured in the presence of EET. In contrast PPAR $\gamma$ and $\mathrm{C} / \mathrm{EBP} \alpha$ protein pattern was the reverse by inhibition of HO-1. PPAR $\gamma$ and $\mathrm{C} / \mathrm{EBP} \alpha$ levels were significantly increased, while $\mathrm{Wnt} / \beta$-catenin protein levels were decreased when compared with adipocytes cultured in the presence of EET. EET-agonist-treated cells showed an increase in $\beta$-catenin. HO- 1 expression was greatly diminished during adipogenic differentiation of MSCs, while FAS and
PPAR $\gamma$ levels increased. Ectopic expression and activation of PPAR $y$ are sufficient to induce adipocyte differentiation [33]. FAS mRNA levels increased dramatically during 3T3-L1 adipocyte differentiation [55]. In agreement with previous studies, Wnt stimulation facilitates disruption of the axinbased complex $[56,57]$. This results in a decrease in the phosphorylation of $\beta$-catenin, which enhances $\beta$-catenin accumulation and activation leading to an arrest in adipogenesis at the early progenitor stage through the blocking of PPAR $\gamma$ signaling $[58,59]$. Furthermore, EETs inhibited MSC-derived stem cell adipogenesis presumably through activation of $\mathrm{HO}-1 / \mathrm{Wnt} / \beta$-catenin and the expression of $\mathrm{C} / \mathrm{EBP} \alpha$, a marker of adipocyte differentiation [54]. In the 

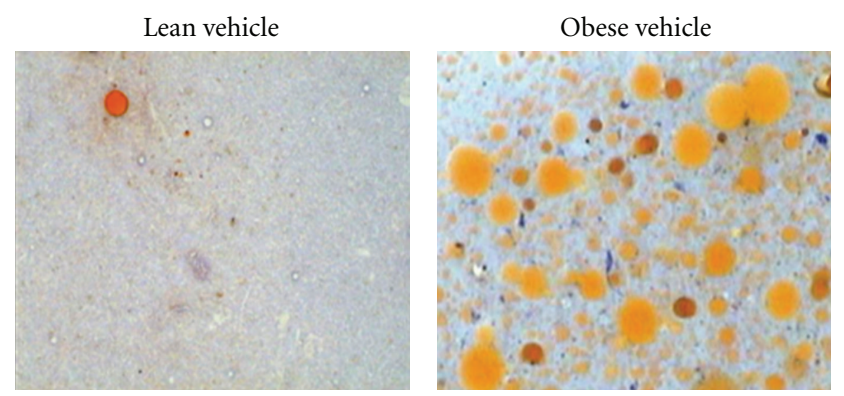

(a)

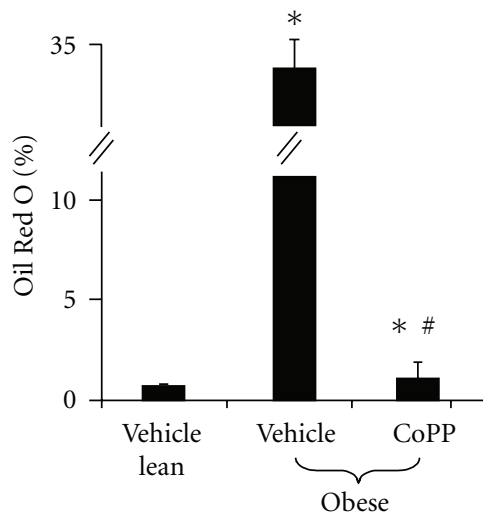

(b)

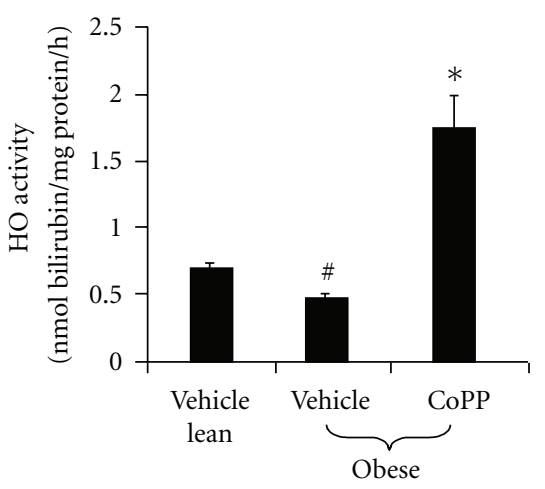

(c)
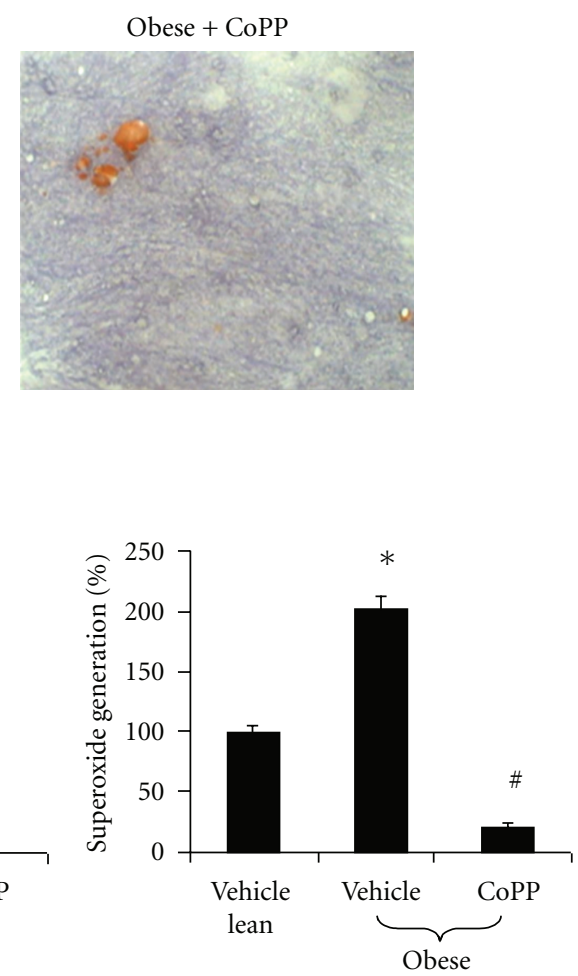

(d)

FIGURE 3: Evaluation of lipid content measured as percentage of Oil Red O staining. (a) Representative photographs demonstrating an increase in lipid droplets in the bone marrow of obese mice compared to lean mice and obese mice treated with CoPP. (b) Quantitative analysis of bone marrow lipid content showing a significant increase in Oil Red O staining in the obese vehicle. Please note bars on the graphs represent the mean \pm SEM of three independent experiments; ${ }^{*} P<0.05$ versus vehicle-treated lean mice; ${ }^{\#} P<0.05$ versus vehicletreated ob mice. (c) HO activity of bone marrow cells showing an increase in HO activity in both lean mice and obese mice treated with $\mathrm{CoPP}$ when compared to obese mice not receiving treatment. Please note bars on the graphs represent the mean \pm SEM of four independent experiments; ${ }^{\#} P<0.05$ versus vehicle-treated lean mice; ${ }^{*} P<0.01$ versus vehicle-treated ob mice. (d) Superoxide generation in bone marrow cells is shown to be increased in obese mice and is lowest in the mice treated with CoPP implying an antioxidant effect of HO. Please note bars on the graphs represent the mean \pm SEM of four independent experiments; ${ }^{*} P<0.05$ obese versus vehicle lean; ${ }^{*} P<0.001$ versus vehicle-treated obese.

present study, the increase of $\mathrm{C} / \mathrm{EBP} \alpha$ was prevented by treatment of an EET agonist at day 10 and day 14. Vanella and coworkers provided direct evidence that EET-agonistinduced activation of $\mathrm{HO}-1$ led to the increase in adiponectin and phosphorylation/inactivation of ACC and consequently decreased of FAS levels.

2.3. Diabetes and HO-1. Diabetes directly affects the integrity and functionality of bone in both humans and animals resulting in osteoporosis and an increase in adipogenesis [60-64]. Patients with diabetes frequently have a lower bone mineral density with associated osteopenia or osteoporosis. Because diabetic individuals commonly have decreased bone mass and bone mineral density, they are more susceptible to fractures and impaired bone healing. This correlation between diabetics and an increase in fractures has been well documented in the literature. A large prospective cohort study of over 32,000 postmenopausal women found that those with type 1 diabetes mellitus were 12 times more likely to experience hip fractures than those women without type 1 diabetes. Women with type 2 diabetes had a 1.7-fold increase in hip fractures when compared to women without this disease [65]. Prevention at decreasing osteoporotic fractures in patients with diabetes can involve strict glucose control, prevention and treatment of vascular complications, regular exercise, and fall prevention [66]. Additionally, biochemical markers of bone turnover are negatively affected in diabetics [67]. One such biomechanical marker, osteocalcin, has been found to be decreased in patients with diabetes [68]. However, the exact pathogenesis of the reduction in bone mass seen in diabetics is unknown. Also, the effect of osteoblast stem cell differentiation under high glucose conditions has not been fully elucidated although, as previously stated, strong evidence supports the belief that hyperglycemic environments decrease osteoblast differentiation and increase adipocyte differentiation [4, 13]. Upregulation of HO-1 expression in obesity and type 2 diabetes results in a decrease in visceral and subcutaneous fat content, improved insulin sensitivity, and increased insulin receptor phosphorylation [22,69-71]. MRI studies showed that upregulation of HO-1 decreased adiposity and adipocyte hypertrophy $[17,70]$. The decrease in HO-1 expression was 

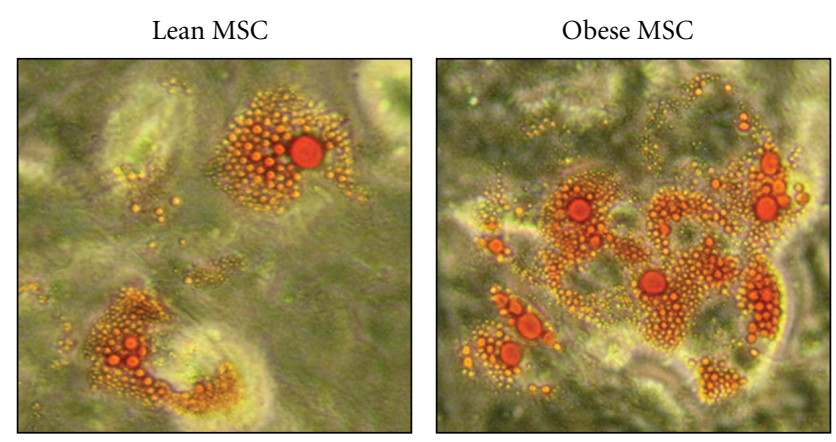

(a)

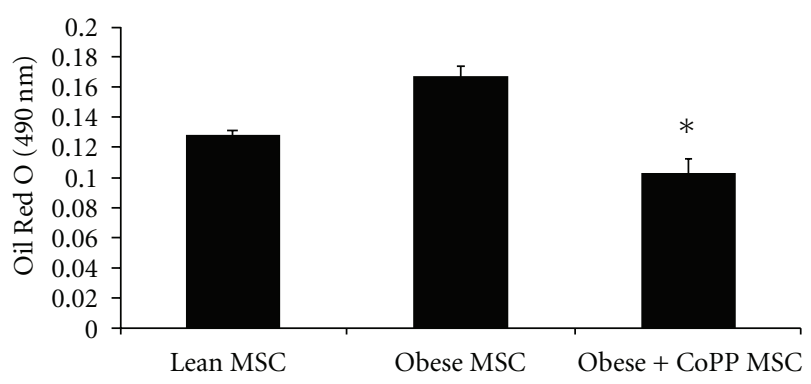

(b)

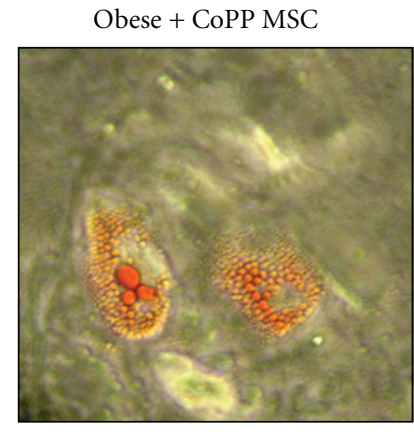

Obese + CoPP MSC (1) 


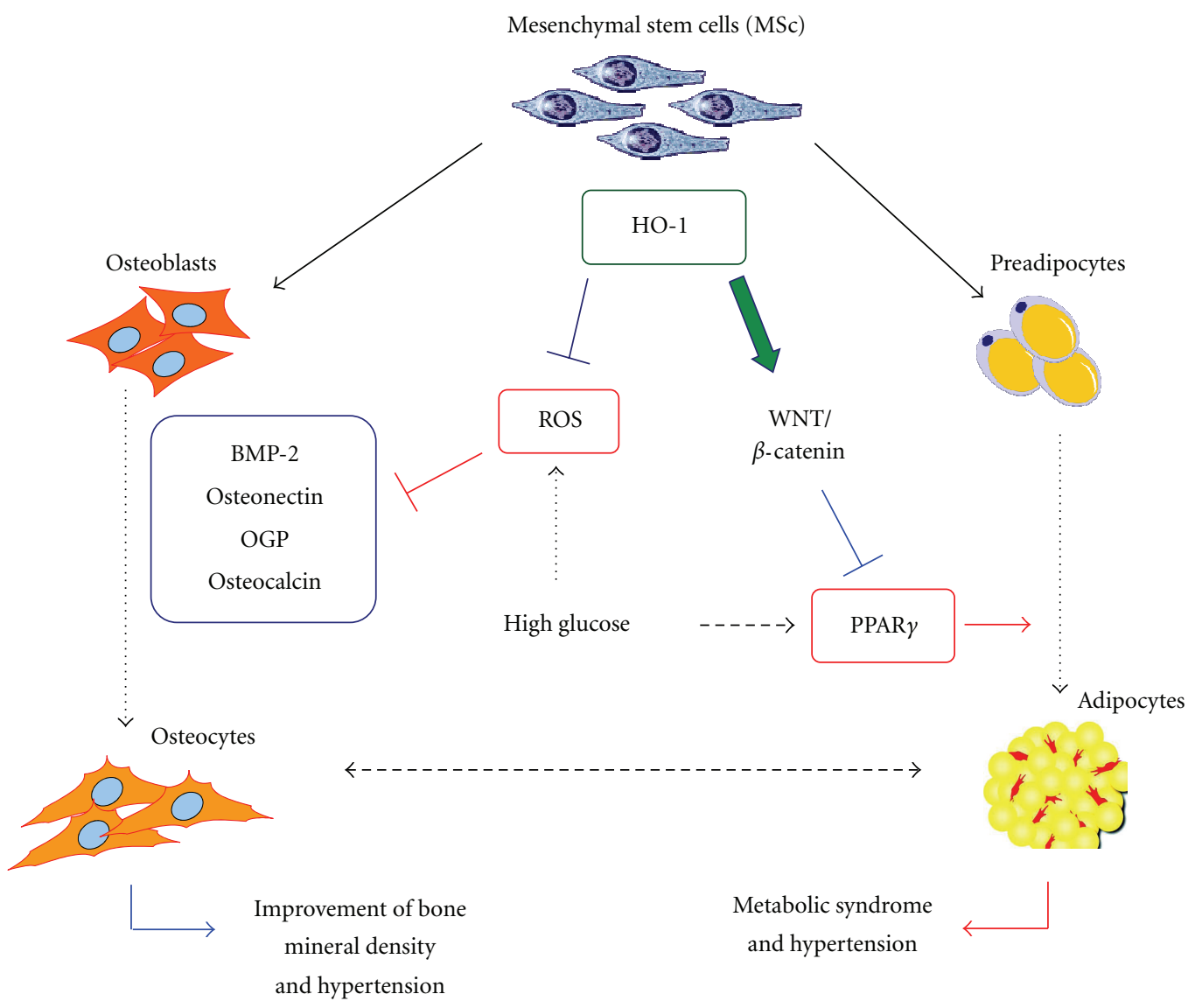

FIGURE 5: Schematic demonstrating the interplay between HO-1, hyperglycemia, ROS, and PPAR- $\gamma$ on the regulation of osteoblast and adipocyte differentiation from mesenchymal stem cells.

found to be reversed by upregulation of HO-1 [23]. Elevated glucose levels cause glucose oxidation which leads to an increase of ROS in endothelial cells. This formation of ROS is believed to be the major factor in endothelial dysfunction such as abnormalities in cell cycling and delayed replication. However, these abnormalities are able to be reversed by antioxidant agents and an increased expression of antioxidant enzymes. Overexpression of HO-1 has been shown to make cells resistant to oxidative stress-causing agents while enhancing cell growth and angiogenesis. Such findings further demonstrate the important cytoprotective and antioxidant role of HO-1. Strong evidence exists that diabetic-induced hyperglycemia has a substantial impact on the development and progression of osteoporosis. The stimulation of osteoblast apoptosis as well as the decrease in osteoblast and increase in adipocyte differentiation all contribute to the decreased bone mineral density observed in individuals with diabetes.

2.4. Future Perspectives. Osteoporosis is a complex condition with significant morbidity, characterized by low bone mass, increased fragility, and fracture risk. Although mechanisms are unclear, there is significant evidence showing the interplay between HO-1, PPAR $\gamma$, oxidative stress, and hyperglycemia. All have a role in the regulation of mesenchymal stem cell differentiation and the development of osteoporosis (Figure 5). Several studies have shown that HO-1 plays a considerable role in reducing oxidative stress, cellular apoptosis, increasing osteoblast differentiation, while simultaneously suppressing adipocyte differentiation from mesenchymal stem cells. HO-1 upregulation causes an increase in alkaline phosphatase and BMP-2 expression and a decrease in PPAR $y$ expression, increasing bone formation and decreasing adipocyte differentiation. Conversely, HO-1 suppression upregulates PPAR $y$ and increases adipogenesis, worse in diabetics, with reduced bone mineral density. Pharmacological and genetic approaches to deliver HO- 1 or products of heme degradation remain to be elucidated for the causes, treatment, and prevention of osteoporosis.

\section{Abbreviations}

HO-1: Heme oxygenase-1

MSCs: Mesenchymal stem cells

PPAR $y$ : Peroxisome proliferator-activated receptor gamma

GSK3 $\beta$ : Glycogen synthase kinase $3 \beta$

aP2: $\quad$ Fatty-acid-binding protein 4 (FABP4)

EGF: Endothelial growth factor

Wnts: Wingless-type 
C/EBP $\alpha$ : Adipogenic transcription factors CCAAT/enhancer binding protein $\alpha$

ROS: $\quad$ Reactive oxygen species

NO: $\quad$ Nitric oxide

BMP-2: Bone morphogenic protein-2

RUNX-2: Runt-related transcription factor 2

OGP: Osteogenic growth peptide

P450: Cytochrome P450

EET: Epoxyeicosatrienoic acids

TZD: Thiazolidinediones

CoPP: Co-protoporphyrin IX

SnMP: Tin-mesoporphyrin IX

AP: $\quad$ Alkaline phosphatase

VEGF: Vascular endothelial growth factor.

\section{Acknowledgments}

This work was supported by National Institutes of Health grants DK-068134, HL-55601 and HL-34300 (Nader G. Abraham). This research was also supported by a grant from the Beatrice Renfield Foundation (Attallah Kappas).

\section{References}

[1] M. F. Pittenger, A. M. Mackay, S. C. Beck et al., "Multilineage potential of adult human mesenchymal stem cells," Science, vol. 284, no. 5411, pp. 143-147, 1999.

[2] G. Ferrari, G. Cusella-De Angelis, M. Coletta et al., "Muscle regeneration by bone marrow-derived myogenic progenitors," Science, vol. 279, no. 5356, pp. 1528-1530, 1998.

[3] I. Barbagallo, D. Tibullo, M. Di Rosa et al., "A cytoprotective role for the heme oxygenase-1/CO pathway during neural differentiation of human mesenchymal stem cells," Journal of Neuroscience Research, vol. 86, no. 9, pp. 1927-1935, 2008.

[4] I. Barbagallo, A. Vanella, S. J. Peterson et al., "Overexpression of heme oxygenase-1 increases human osteoblast stem cell differentiation," Journal of Bone and Mineral Metabolism, vol. 28, no. 3, pp. 276-288, 2010.

[5] Z. X. Chen, M. Chang, Y. L. Peng et al., "Osteogenic growth peptide C-terminal pentapeptide [OGP(10-14)] acts on rat bone marrow mesenchymal stem cells to promote differentiation to osteoblasts and to inhibit differentiation to adipocytes," Regulatory Peptides, vol. 142, no. 1-2, pp. 16-23, 2007.

[6] A. Spreafico, B. Frediani, C. Capperucci et al., "Osteogenic growth peptide effects on primary human osteoblast cultures: potential relevance for the treatment of glucocorticoidinduced osteoporosis," Journal of Cellular Biochemistry, vol. 98, no. 4, pp. 1007-1020, 2006.

[7] R. S. Weinstein, J. R. Chen, C. C. Powers et al., "Promotion of osteoclast survival and antagonism of bisphosphonateinduced osteoclast apoptosis by glucocorticoids," The Journal of Clinical Investigation, vol. 109, no. 8, pp. 1041-1048, 2002.

[8] N. G. Abraham, "Molecular regulation-biological role of heme in hematopoiesis," Blood Reviews, vol. 5, no. 1, pp. 1928, 1991.

[9] S. Khosla and B. L. Riggs, "Pathophysiology of age-related bone loss and osteoporosis," Endocrinology and Metabolism Clinics of North America, vol. 34, no. 4, pp. 1015-1030, 2005.

[10] S. C. Manolagas and A. M. Parfitt, "What old means to bone,"
Trends in Endocrinology and Metabolism, vol. 21, no. 6, pp. 369-374, 2010.

[11] E. S. Strotmeyer, J. A. Cauley, A. V. Schwartz et al., "Nontraumatic fracture risk with diabetes mellitus and impaired fasting glucose in older white and black adults: the health, aging, and body composition study," Archives of Internal Medicine, vol. 165, no. 14, pp. 1612-1617, 2005.

[12] N. G. Abraham, J. D. Lutton, and R. D. Levere, "Heme metabolism and erythropoiesis in abnormal iron states: role of $\delta$-aminolevulinic acid synthase and heme oxygenase," Experimental Hematology, vol. 13, no. 8, pp. 838-843, 1985.

[13] L. Vanella, D. H. Kim, D. Asprinio et al., "HO-1 expression increases mesenchymal stem cell-derived osteoblasts but decreases adipocyte lineage," Bone, vol. 46, no. 1, pp. 236-243, 2010.

[14] K. E. Armour, K. J. Armour, M. E. Gallagher et al., "Defective bone formation and anabolic response to exogenous estrogen in mice with targeted disruption of endothelial nitric oxide synthase," Endocrinology, vol. 142, no. 2, pp. 760-766, 2001.

[15] G. Mundy, R. Garrett, S. Harris et al., "Stimulation of bone formation in vitro and in rodents by statins," Science, vol. 286, no. 5446, pp. 1946-1949, 1999.

[16] I. R. Garrett, G. Gutierrez, and G. R. Mundy, "Statins and bone formation," Current Pharmaceutical Design, vol. 7, no. 8, pp. 715-736, 2001.

[17] S. J. Peterson, D. H. K. Kim, M. Li et al., "The L-4F mimetic peptide prevents insulin resistance through increased levels of HO-1, pAMPK, and pAKT in obese mice," Journal of Lipid Research, vol. 50, no. 7, pp. 1293-1304, 2009.

[18] A. L. Kruger, S. J. Peterson, M. L. Schwartzman et al., "Upregulation of heme oxygenase provides vascular protection in an animal model of diabetes through its antioxidant and antiapoptotic effects," Journal of Pharmacology and Experimental Therapeutics, vol. 319, no. 3, pp. 1144-1152, 2006.

[19] S. Quan, P. M. Kaminski, L. Yang et al., "Heme oxygenase-1 prevents superoxide anion-associated endothelial cell sloughing in diabetic rats," Biochemical and Biophysical Research Communications, vol. 315, no. 2, pp. 509-516, 2004.

[20] S. H. Chang, J. Garcia, J. A. Melendez, M. S. Kilberg, and A. Agarwal, "Haem oxygenase 1 gene induction by glucose deprivation is mediated by reactive oxygen species via the mitochondrial electron-transport chain," Biochemical Journal, vol. 371, no. 3, pp. 877-885, 2003.

[21] N. G. Abraham, T. Kushida, J. McClung et al., "Heme oxygenase-1 attenuates glucose-mediated cell growth arrest and apoptosis in human microvessel endothelial cells," Circulation Research, vol. 93, no. 6, pp. 507-514, 2003.

[22] M. Li, D. H. Kim, P. L. Tsenovoy et al., "Treatment of obese diabetic mice with a heme oxygenase inducer reduces visceral and subcutaneous adiposity, increases adiponectin levels, and improves insulin sensitivity and glucose tolerance," Diabetes, vol. 57, no. 6, pp. 1526-1535, 2008.

[23] S. Turkseven, A. Kruger, C. J. Mingone et al., "Antioxidant mechanism of heme oxygenase-1 involves an increase in superoxide dismutase and catalase in experimental diabetes," American Journal of Physiology, vol. 289, no. 2, pp. H701H707, 2005.

[24] M. A. Di Noia, S. Van Driesche, F. Palmieri et al., "Heme oxygenase-1 enhances renal mitochondrial transport carriers and cytochrome c oxidase activity in experimental diabetes," The Journal of Biological Chemistry, vol. 281, no. 23, pp. 15687-15693, 2006. 
[25] M. A. Puertollano, M. A. de Pablo, and G. Álvarez de Cienfuegos, "Anti-oxidant properties of $\mathrm{N}$-acetyl-L-cysteine do not improve the immune resistance of mice fed dietary lipids to Listeria monocytogenes infection," Clinical Nutrition, vol. 22, no. 3, pp. 313-319, 2003.

[26] L. Mueller and V. Boehm, "Antioxidant activity of $\beta$-carotene compounds in different in vitro assays," Molecules, vol. 16, no. 2, pp. 1055-1069, 2011.

[27] M. Tiedge, S. Lortz, J. Drinkgern, and S. Lenzen, "Relation between antioxidant enzyme gene expression and antioxidative defense status of insulin-producing cells," Diabetes, vol. 46, no. 11, pp. 1733-1742, 1997.

[28] B. Larsson, "Obesity, fat distribution and cardiovascular disease," International Journal of Obesity, vol. 15, supplement 2, pp. 53-57, 1991.

[29] J. E. Hall, “The kidney, hypertension, and obesity," Hypertension, vol. 41, no. 3, pp. 625-633, 2003.

[30] A. Pileggi, R. Damaris Molano, T. Berney et al., "Heme oxygenase-1 induction in islet cells results in protection from apoptosis and improved in vivo function after transplantation," Diabetes, vol. 50, no. 9, pp. 1983-1991, 2001.

[31] S. Brouard, L. E. Otterbein, J. Anrather et al., "Carbon monoxide generated by heme oxygenase 1 suppresses endothelial cell apoptosis," Journal of Experimental Medicine, vol. 192, no. 7, pp. 1015-1025, 2000.

[32] S. E. Schadinger, N. L. R. Bucher, B. M. Schreiber, and S. R. Farmer, "PPAR $\gamma 2$ regulates lipogenesis and lipid accumulation in steatotic hepatocytes," American Journal of Physiology, vol. 288, no. 6, pp. E1195-E1205, 2005.

[33] J. Liu and S. R. Farmer, "Regulating the balance between peroxisome proliferator-activated receptor $\gamma$ and $\beta$-catenin signaling during adipogenesis: a glycogen synthase kinase $3 \beta$ phosphorylation-defective mutant of $\beta$-catenin inhibits expression of a subset of adipogenic genes," The Journal of Biological Chemistry, vol. 279, no. 43, pp. 45020-45027, 2004.

[34] O. Gavrilova, M. Haluzik, K. Matsusue et al., "Liver peroxisome proliferator-activated receptor gamma contributes to hepatic steatosis, triglyceride clearance, and regulation of body fat mass," The Journal of Biological Chemistry, vol. 278, no. 36, pp. 34268-34276, 2003.

[35] K. Matsusue, M. Haluzik, G. Lambert et al., "Liver-specific disruption of PPAR $\gamma$ in leptin-deficient mice improves fatty liver but aggravates diabetic phenotypes," The Journal of Clinical Investigation, vol. 111, no. 5, pp. 737-747, 2003.

[36] N. Kubota, Y. Terauchi, H. Miki et al., "PPAR $\gamma$ mediates high-fat diet-induced adipocyte hypertrophy and insulin resistance," Molecular Cell, vol. 4, no. 4, pp. 597-609, 1999.

[37] T. Akune, S. Ohba, S. Kamekura et al., "PPAR $\gamma$ insufficiency enhances osteogenesis through osteoblast formation from bone marrow progenitors," The Journal of Clinical Investigation, vol. 113, no. 6, pp. 846-855, 2004.

[38] B. Lecka-Czernik, I. Gubrij, E. J. Moerman et al., "Inhibition of Osf2/Cbfa1 expression and terminal osteoblast differentiation by PPAR $\gamma 2$," Journal of Cellular Biochemistry, vol. 74, no. 3, pp. 357-371, 1999.

[39] E. D. Rosen and B. M. Spiegelman, "PPARgamma: a nuclear regulator of metabolism, differentiation, and cell growth," The Journal of Biological Chemistry, vol. 276, no. 41, pp. 3773137734, 2001.

[40] O. Braissant, F. Foufelle, C. Scotto, M. Dauça, and W. Wahli, "Differential expression of peroxisome proliferator-activated receptors (PPARs): tissue distribution of PPAR- $\alpha,-\beta$, and $-\gamma$ in the adult rat," Endocrinology, vol. 137, no. 1, pp. 354-366, 1996.
[41] S. G. Moore and K. L. Dawson, "Red and yellow marrow in the femur: age-related changes in appearance at MR imaging," Radiology, vol. 175, no. 1, pp. 219-223, 1990.

[42] J. M. Gimble, C. E. Robinson, X. Wu, and K. A. Kelly, "The function of adipocytes in the bone marrow stroma: an update," Bone, vol. 19, no. 5, pp. 421-428, 1996.

[43] M. Kawai, K. M. Sousa, O. A. MacDougald, and C. J. Rosen, "The many facets of PPAR $\gamma$ : novel insights for the skeleton," American Journal of Physiology, vol. 299, no. 1, pp. E3-E9, 2010.

[44] M. A. Sorocéanu, D. Miao, X. Y. Bai, H. Su, D. Goltzman, and A. C. Karaplis, "Rosiglitazone impacts negatively on bone by promoting osteoblast/osteocyte apoptosis," Journal of Endocrinology, vol. 183, no. 1, pp. 203-216, 2004.

[45] G. Mabilleau, A. Mieczkowska, and M. E. Edmonds, "Thiazolidinediones induce osteocyte apoptosis and increase sclerostin expression," Diabetic Medicine, vol. 27, no. 8, pp. 925932, 2010.

[46] A. K. McDonough, R. S. Rosenthal, X. Cao, and K. G. Saag, "The effect of thiazolidinediones on BMD and osteoporosis," Nature Clinical Practice Endocrinology and Metabolism, vol. 4, no. 9, pp. 507-513, 2008.

[47] K. R. Shockley, O. P. Lazarenko, P. J. Czernik, C. J. Rosen, G. A. Churchill, and B. Lecka-Czernik, "PPAR $\gamma 2$ nuclear receptor controls multiple regulatory pathways of osteoblast differentiation from marrow mesenchymal stem cells," Journal of Cellular Biochemistry, vol. 106, no. 2, pp. 232-246, 2009.

[48] B. Lecka-Czernik, C. Ackert-Bicknell, M. L. Adamo et al., "Activation of peroxisome proliferator-activated receptor $\gamma$ $(\operatorname{PPAR} \gamma)$ by rosiglitazone suppresses components of the insulin-like growth factor regulatory system in vitro and in vivo," Endocrinology, vol. 148, no. 2, pp. 903-911, 2007.

[49] A. V. Schwartz, D. E. Sellmeyer, E. Vittinghoff et al., "Thiazolidinedione use and bone loss in older diabetic adults," Journal of Clinical Endocrinology and Metabolism, vol. 91, no. 9, pp. 3349-3354, 2006.

[50] B. Lecka-Czernik, "Bone as a target of type 2 diabetes treatment," Current Opinion in Investigational Drugs, vol. 10, no. 10, pp. 1085-1090, 2009.

[51] Z. A. Habib, S. L. Havstad, K. Wells, G. Divine, M. Pladevall, and L. K. Williams, "Thiazolidinedione use and the longitudinal risk of fractures in patients with type 2 diabetes mellitus," Journal of Clinical Endocrinology and Metabolism, vol. 95, no. 2, pp. 592-600, 2010.

[52] A. Grey, M. Bolland, G. Gamble et al., "The peroxisome proliferator-activated receptor- $\gamma$ agonist rosiglitazone decreases bone formation and bone mineral density in healthy postmenopausal women: a randomized, controlled trial," Journal of Clinical Endocrinology and Metabolism, vol. 92, no. 4, pp. 1305-1310, 2007.

[53] M. Kawai and C. J. Rosen, "PPAR $\gamma$ : a circadian transcription factor in adipogenesis and osteogenesis," Nature Reviews Endocrinology, vol. 6, no. 11, pp. 629-636, 2010.

[54] L. Vanella, D. H. Kim, K. Sodhi et al., "Crosstalk between EET and HO-1 downregulates Bach1 and adipogenic marker expression in mesenchymal stem cell derived adipocytes," Prostaglandins and Other Lipid Mediators, vol. 96, no. 1-4, pp. 54-62, 2011.

[55] N. Moustaid and H. S. Sul, "Regulation of expression of the fatty acid synthase gene in 3T3-L1 cells by differentiation and triiodothyronine," The Journal of Biological Chemistry, vol. 266, no. 28, pp. 18550-18554, 1991.

[56] R. S. Jope and G. V. W. Johnson, "The glamour and gloom of glycogen synthase kinase-3," Trends in Biochemical Sciences, 
vol. 29, no. 2, pp. 95-102, 2004.

[57] S. L. Etheridge, G. J. Spencer, D. J. Heath, and P. G. Genever, "Expression profiling and functional analysis of Wnt signaling mechanisms in mesenchymal stem cells," Stem Cells, vol. 22, no. 5, pp. 849-860, 2004.

[58] S. E. Ross, N. Hemati, K. A. Longo et al., "Inhibition of adipogenesis by Wnt signaling," Science, vol. 289, no. 5481, pp. 950-953, 2000.

[59] M. Okamura, H. Kudo, K. I. Wakabayashi et al., "COUP-TFII acts downstream of Wnt/ $\beta$-catenin signal to silence PPAR $\gamma$ gene expression and repress adipogenesis," Proceedings of the National Academy of Sciences of the United States of America, vol. 106, no. 14, pp. 5819-5824, 2009.

[60] P. Tontonoz, E. Hu, R. A. Graves, A. I. Budavari, and B. M. Spiegelman, "mPPAR $\gamma 2$ : tissue-specific regulator of an adipocyte enhancer," Genes and Development, vol. 8, no. 10, pp. 1224-1234, 1994.

[61] E. J. Hamilton, V. Rakic, W. A. Davis et al., "Prevalence and predictors of osteopenia and osteoporosis in adults with Type 1 diabetes," Diabetic Medicine, vol. 26, no. 1, pp. 45-52, 2009.

[62] J. L. Fowlkes, R. C. Bunn, L. Liu et al., "Runt-related transcription factor 2 (RUNX2) and RUNX2-related osteogenic genes are down-regulated throughout osteogenesis in type 1 diabetes mellitus," Endocrinology, vol. 149, no. 4, pp. 1697-1704, 2008.

[63] A. V. Schwartz, "Diabetes mellitus: does it affect bone?" Calcified Tissue International, vol. 73, no. 6, pp. 515-519, 2003.

[64] A. V. Schwartz, D. E. Sellmeyer, K. E. Ensrud et al., "Older women with diabetes have an increased risk of fracture: a prospective study," Journal of Clinical Endocrinology and Metabolism, vol. 86, no. 1, pp. 32-38, 2001.

[65] K. K. Nicodemus and A. R. Folsom, "Type 1 and type 2 diabetes and incident hip fractures in postmenopausal women," Diabetes Care, vol. 24, no. 7, pp. 1192-1197, 2001.

[66] N. K. Lee, H. Sowa, E. Hinoi et al., "Endocrine regulation of energy metabolism by the skeleton," Cell, vol. 130, no. 3, pp. 456-469, 2007.

[67] S. Yaturu, S. Humphrey, C. Landry, and S. K. Jain, "Decreased bone mineral density in men with metabolic syndrome alone and with type 2 diabetes," Medical Science Monitor, vol. 15, no. 1, pp. CR5-CR9, 2009.

[68] H. He, R. Liu, T. Desta, C. Leone, L. C. Gerstenfeld, and D. T. Graves, "Diabetes causes decreased osteoclastogenesis, reduced bone formation, and enhanced apoptosis of osteoblastic cells in bacteria stimulated bone loss," Endocrinology, vol. 145, no. 1, pp. 447-452, 2004.

[69] H. K. Dong, A. P. Burgess, M. Li et al., "Heme oxygenasemediated increases in adiponectin decrease fat content and inflammatory cytokines tumor necrosis factor- $\alpha$ and interleukin-6 in Zucker rats and reduce adipogenesis in human mesenchymal stem cells," Journal of Pharmacology and Experimental Therapeutics, vol. 325, no. 3, pp. 833-840, 2008.

[70] A. Nicolai, M. Li, D. H. Kim et al., "Heme oxygenase1 induction remodels adipose tissue and improves insulin sensitivity in obesity-induced diabetic rats," Hypertension, vol. 53, no. 3, pp. 508-515, 2009.

[71] S. J. Peterson, G. Drummond, D. H. Kim et al., "L-4F treatment reduces adiposity, increases adiponectin levels, and improves insulin sensitivity in obese mice," Journal of Lipid Research, vol. 49, no. 8, pp. 1658-1669, 2008.

[72] A. Burgess, M. Li, L. Vanella et al., "Adipocyte heme oxygenase-1 induction attenuates metabolic syndrome in both male and female obese mice," Hypertension, vol. 56, no. 6, pp. 1124-1130, 2010.

[73] K. Sodhi, K. Inoue, K. H. Gotlinger et al., "Epoxyeicosatrienoic acid agonist rescues the metabolic syndrome phenotype of HO-2-null mice," Journal of Pharmacology and Experimental Therapeutics, vol. 331, no. 3, pp. 906-916, 2009.

[74] J. Cao, K. Sodhi, K. Inoue et al., "Lentiviral-human heme oxygenase targeting endothelium improved vascular function in angiotensin II animal model of hypertension," Human Gene Therapy, vol. 22, no. 3, pp. 271-282, 2011.

[75] D. Sacerdoti, M. Bolognesi, M. Di Pascoli et al., "Rat mesenteric arterial dilator response to 11,12-epoxyeicosatrienoic acid is mediated by activating heme oxygenase," American Journal of Physiology, vol. 291, no. 4, pp. H1999-H2002, 2006.

[76] D. Sacerdoti, C. Colombrita, M. Di Pascoli et al., "11,12Epoxyeicosatrienoic acid stimulates heme-oxygenase-1 in endothelial cells," Prostaglandins and Other Lipid Mediators, vol. 82, no. 1-4, pp. 155-161, 2007.

[77] M. L. Schwartzman, P. Iserovich, K. Gotlinger et al., "Profile of lipid and protein autacoids in diabetic vitreous correlates with the progression of diabetic retinopathy," Diabetes, vol. 59, no. 7, pp. 1780-1788, 2010.

[78] P. Luo and M.-H. Wang, "Eicosanoids, $\beta$-cell function, and diabetes," Prostaglandins and Other Lipid Mediators, vol. 95, no. 1-4, pp. 1-10, 2011.

[79] D. H. Kim, L. Vanella, K. Inoue et al., "Epoxyeicosatrienoic acid agonist regulates human mesenchymal stem cell-derived adipocytes through activation of HO-1-pAKT signaling and a decrease in PPAR $\gamma$," Stem Cells and Development, vol. 19, no. 12, pp. 1863-1873, 2010.

[80] A. L. Kruger, S. Peterson, S. Turkseven et al., "D-4F induces heme oxygenase-1 and extracellular superoxide dismutase, decreases endothelial cell sloughing, and improves vascular reactivity in rat model of diabetes," Circulation, vol. 111, no. 23, pp. 3126-3134, 2005.

[81] R. Mishra, S. N. Emancipator, T. Kern, and M. S. Simonson, "High glucose evokes an intrinsic proapoptotic signaling pathway in mesangial cells," Kidney International, vol. 67, no. 1, pp. 82-93, 2005. 


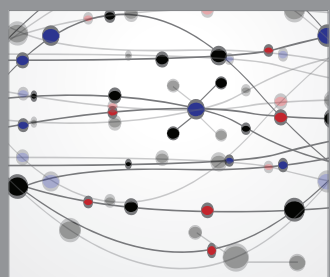

The Scientific World Journal
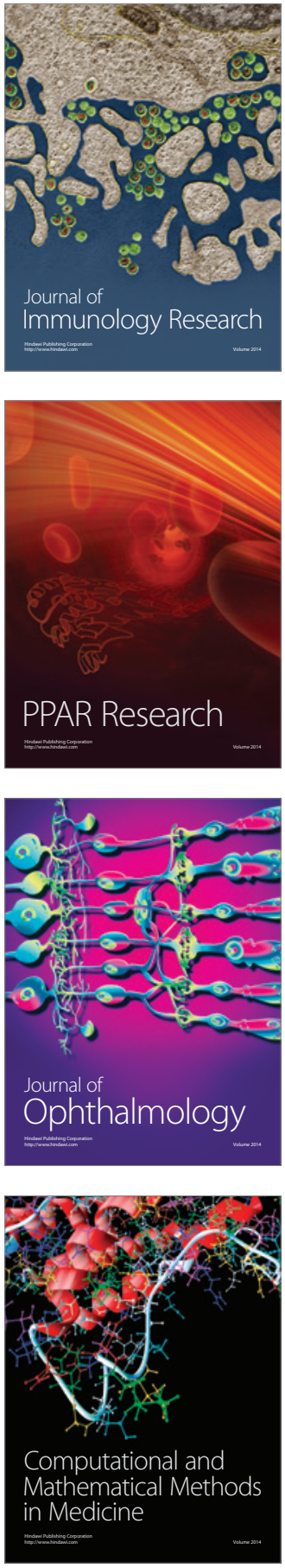

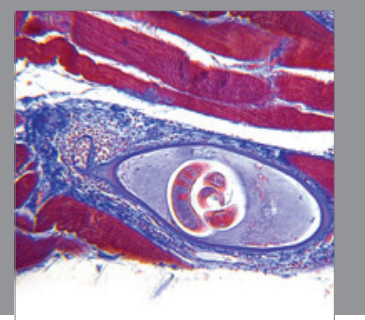

Gastroenterology

Research and Practice
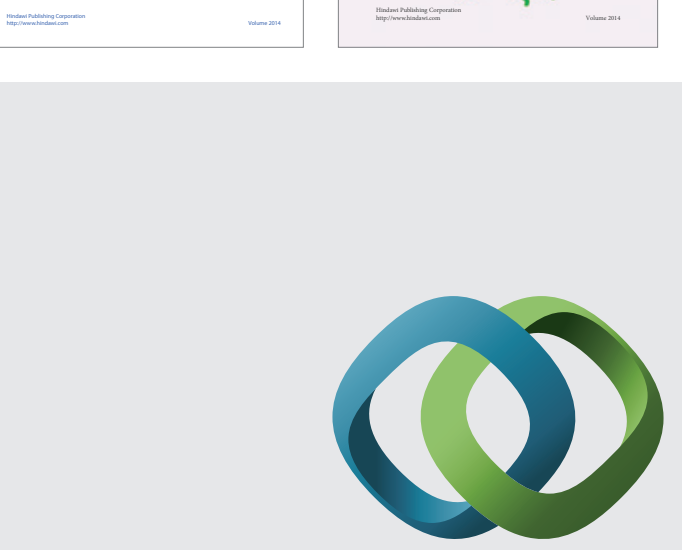

\section{Hindawi}

Submit your manuscripts at

http://www.hindawi.com
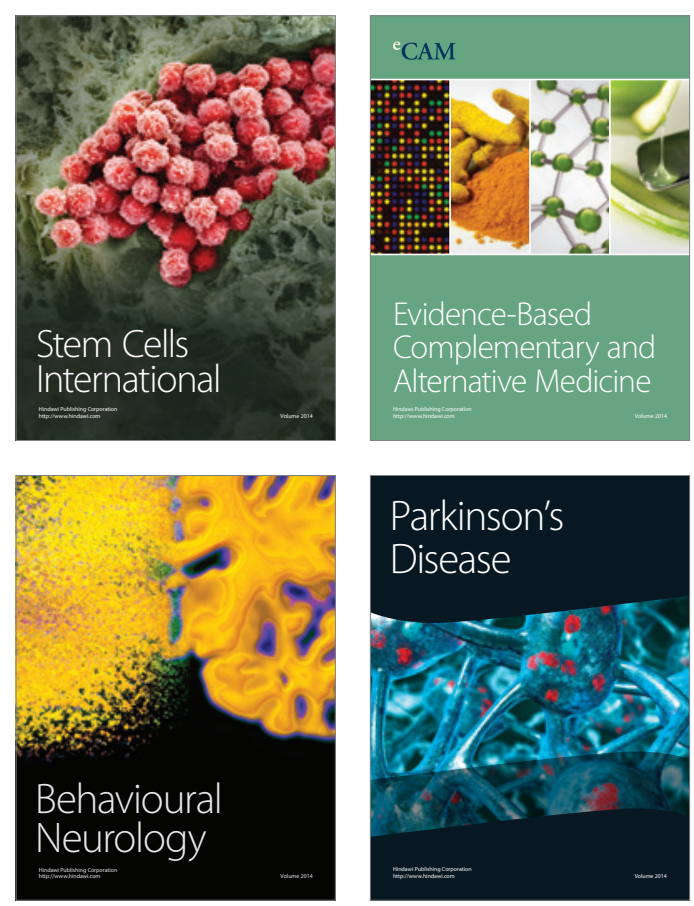

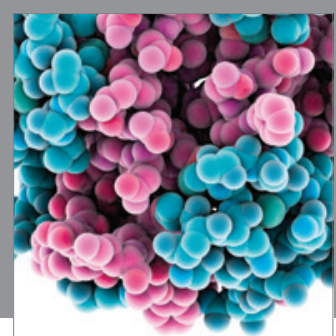

Journal of
Diabetes Research

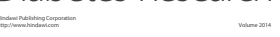

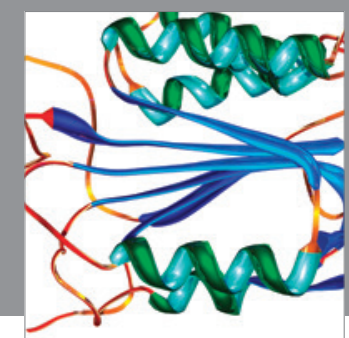

Disease Markers
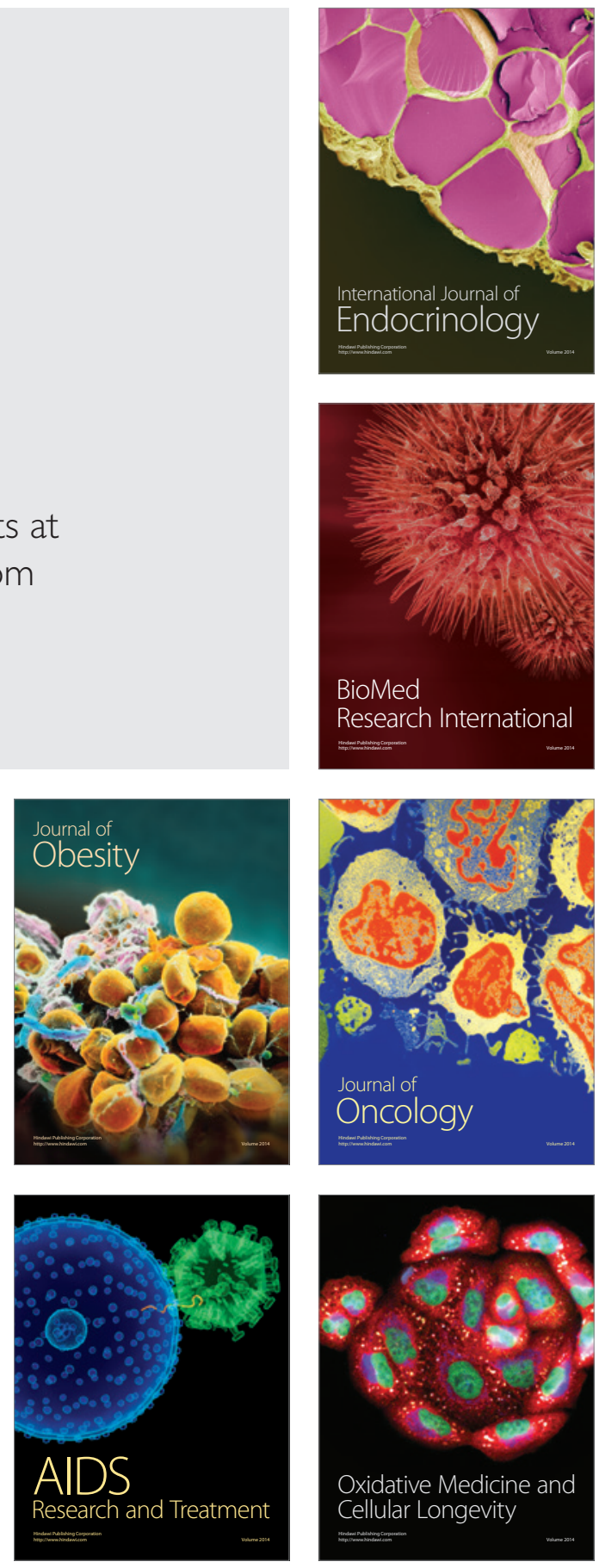